\title{
Effect of non-standard neutrino interactions on the sensitivities of DUNE
}

\author{
K. N. Deepthi* \\ Physical Research Laboratory, Ahmedabad 380 009, India \\ E-mail: deepthieprl.res.in
}

\section{Srubabati Goswami}

Physical Research Laboratory, Ahmedabad 380 009, India

E-mail: sruba@prl.res.in

\section{Newton Nath}

Physical Research Laboratory, Ahmedabad 380 009, India

Indian Institute of Technology, Ahmedabad-382424, India

E-mail: newtoneprl.res.in

\begin{abstract}
In this work, we consider the effect of non-standard interactions (NSIs) on the propagation of neutrinos through matter and how they in turn affect the sensitivity of the Deep Underground Neutrino Experiment (DUNE) in determining the mass hierarchy. We emphasize on the special case - when the diagonal NSI parameter $\varepsilon_{e e}=-1$ at which the standard matter effect gets nullified. We show that, if in addition, there is maximal $\mathrm{CP}$ violation then this gives rise to an exact intrinsic hierarchy degeneracy, in the appearance channel, irrespective of the baseline and energy. We learn that the introduction of the off-diagonal NSI parameter, $\varepsilon_{e \tau}$, shifts the position of the intrinsic degeneracy to a different $\varepsilon_{e e}$. Moreover the unknown magnitude and phases of the off-diagonal NSI parameters can give additional degeneracies. Considering current model independent bounds on NSI parameters we observe that the hierarchy sensitivity of DUNE gets seriously compromised if NSI exists in nature. A signal of neutrino mass hierarchy at DUNE will therefore be able to rule out certain ranges of the NSI parameters. Additionally, we discuss the implications of $\varepsilon_{e e}=-1$ (in the Earth) on the MSW effect in the Sun.
\end{abstract}

The 19th International Workshop on Neutrinos from Accelerators-NUFACT2017

25-30 September, 2017

Uppsala University, Uppsala, Sweden

* Speaker. 
Introduction : Neutrino oscillation has been established as the dominant phenomenon to explain the data from various neutrino experiments. However, the possibility of sub-leading effects like non-standard interactions (NSIs) [1,2] originating from new physics beyond the Standard Model (SM) cannot be ignored. In this work, we consider the effect of NSIs on the propagation of neutrinos through matter and how they in turn affect the sensitivity of the Deep Underground Neutrino Experiment (DUNE) in determining the neutrino mass hierarchy.

We study the effect of matter NSI while neglecting the production and detection NSIs as these are bounded by one order of magnitude stronger than the former.

The neutral current NSIs are described by the effective four-fermion Lagrangian density with dimension $d=6$ operators as

$$
\mathscr{L}_{N S I}^{N C}=-2 \sqrt{2} G_{F} \Sigma_{f, \alpha, \beta, P_{C}} \varepsilon_{\alpha \beta}^{f C}\left(\bar{v}_{\alpha} \gamma^{\mu} P_{L} v_{\beta}\right)\left(\bar{f} \gamma_{\mu} P_{C} f\right)+\text { h.c. }
$$

where $\alpha, \beta=e, \mu, \tau, C=L, R$ stands for chirality, $f=u, d, e, \varepsilon_{\alpha \beta}^{f C}$ are the NSI parameters and $G_{F}$ is the Fermi constant.

The effective hamiltonian in the flavor basis can be written as,

$$
H=\frac{1}{2 E}\left[\operatorname{Udiag}\left(0, \Delta m_{21}^{2}, \Delta m_{31}^{2}\right) U^{\dagger}+V\right],
$$

where $U$ is the PMNS mixing matrix which is a function of three mixing angles $\left(\theta_{i j}, i<j=1,2,3\right)$ and a Dirac CP phase $\delta_{C P} . V$ stands for the potential arising from the interactions of neutrinos with matter,

$$
V=2 \sqrt{2} G_{F} N_{e}(r) E\left(\begin{array}{ccc}
1+\varepsilon_{e e} & \varepsilon_{e \mu} e^{i \phi_{e \mu}} & \varepsilon_{e \tau} e^{i \phi_{e \tau}} \\
\varepsilon_{e \mu} e^{-i \phi_{e \mu}} & \varepsilon_{\mu \mu} & \varepsilon_{\mu \tau} e^{i \phi_{\mu \tau}} \\
\varepsilon_{e \tau} e^{-i \phi_{e \tau}} & \varepsilon_{\mu \tau} e^{-i \phi_{\mu \tau}} & \varepsilon_{\tau \tau}
\end{array}\right)
$$

where $\varepsilon_{\alpha \beta} e^{i \phi_{\alpha \beta}} \equiv \sum_{f, C} \varepsilon_{\alpha \beta}^{f C} \frac{N_{f}(r)}{N_{e}(r)}, N_{f}(r)$ is the number density of fermion $f$ at a distance ' $r$ ' in the matter.

For neutral matter (Earth $\varepsilon_{\alpha \beta}^{\oplus}$, Sun $\left.\varepsilon_{\alpha \beta}^{\odot}\right) \varepsilon_{\alpha \beta}$ can be expressed as

$$
\varepsilon_{\alpha \beta}=\varepsilon_{\alpha \beta}^{e}+\left(2+Y_{n}(r)\right) \varepsilon_{\alpha \beta}^{u}+\left(1+2 Y_{n}(r)\right) \varepsilon_{\alpha \beta}^{d}
$$

where $Y_{n}(r)=\frac{N_{n}(r)}{N_{e}(r)}$.

In the presence of only non-zero diagonal NSI, if $\varepsilon_{e e}=-1$ then the standard matter effect is nullified as can be seen from eq. (3). This leads to an intrinsic hierarchy degeneracy in the appearance probability channel $P_{\mu e}$. This degeneracy is found to be independent of baseline and energy. In this work, we have simulated DUNE experiment using General Long Baseline Experiment Simulator (GLoBES) [3, 4] and studied the affect of this degeneracy on its hierarchy sensitivity.

The left panel of fig. (1) shows the behavior of $P_{\mu e}$ as a function of $\varepsilon_{e e}$ for the DUNE baseline of $1300 \mathrm{~km}$ at fixed energy $E=2 \mathrm{GeV}$ and $\delta_{C P}=-\pi / 2$. It can be seen from the figure that at $\varepsilon_{e e}=0$, which corresponds to the standard case, there is a huge separation between $\mathrm{NH}$ and $\mathrm{IH}$ bands arising due to the large matter effect in the DUNE. However, at $\varepsilon_{e e}=-1$ we have $P_{\mu e}^{N H}=P_{\mu e}^{I H}$ leading to an intrinsic hierarchy degeneracy of the form $\mathrm{WH}-\mathrm{RO}-\mathrm{R} \delta_{C P}{ }^{1}$. This is a special case of the generalized degeneracy discussed in [5].

\footnotetext{
${ }^{1}$ Note that $\mathrm{WH}=$ Wrong Hierarchy, $\mathrm{RO}=$ Right Octant, $\mathrm{R} \delta_{C P}=$ Right $\delta_{C P}, \mathrm{~W} \delta_{C P}=$ Wrong $\delta_{C P}$.
} 
In the right panel of fig. (1), we plotted $P_{\mu e}$ vs $\delta_{C P}$ for several values of $\varepsilon_{e e}$. We have observed various degenerate solutions of the form listed below :

1. The WH-RO-R $\delta_{C P}$ occurring at $\delta_{C P}= \pm \pi / 2$ that can be seen by comparing blue and magenta (or the brown and grey) bands. This can be defined as $P_{\mu e}^{N H}\left(\varepsilon_{e e}, \delta_{C P}= \pm \pi / 2\right)=P_{\mu e}^{I H}\left(-\varepsilon_{e e}-\right.$ $\left.2, \delta_{C P}= \pm \pi / 2\right)$ as discussed in the previous plot.

2. When a horizontal line is drawn intersecting the blue band with magenta band or brown band with grey band WH-RO-W $\delta_{C P}$ solutions of the form $P_{\mu e}^{N H}\left(\varepsilon_{e e}, \delta_{C P}\right)=P_{\mu e}^{I H}\left(-\varepsilon_{e e}-2, \delta_{C P}^{\prime}\right)$ occur.

3. A more generalized degeneracy of the form $P\left(\varepsilon_{e e}, \delta_{C P}\right)=P\left(\varepsilon_{e e}^{\prime}, \delta_{C P}^{\prime}\right)$ corresponding to $\varepsilon_{e e} \rightarrow$ $-\varepsilon_{e e}-2$ can also occur. This can be seen from the comparison of the yellow band and the dark-red band.

All the above conclusions also stand true for the appearance channel of anti-neutrinos.
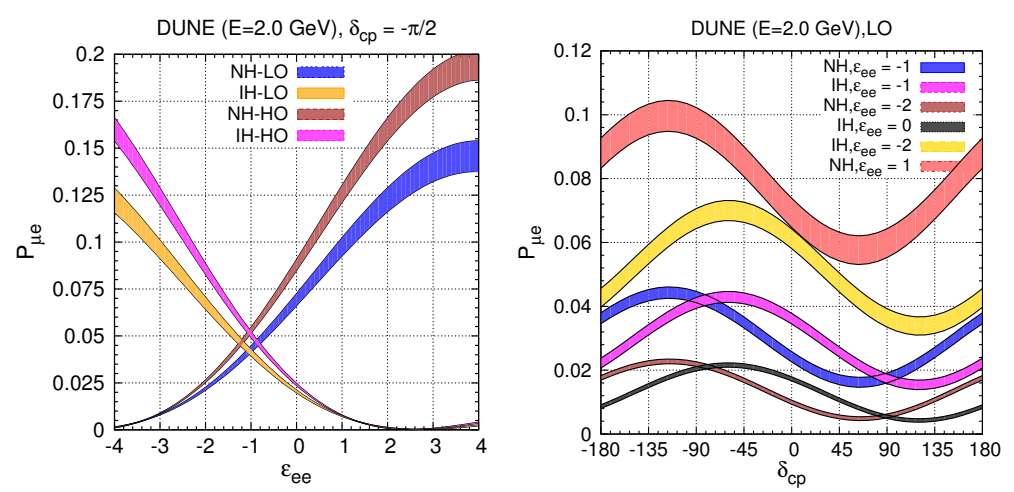

Figure 1: Left panel : $P_{\mu e}$ vs $\varepsilon_{e e}$ for DUNE. The bands are over $\theta_{23}$, for $\operatorname{LO}\left(\theta_{23}=39^{\circ}-42^{\circ}\right)$ and for $\mathrm{HO}\left(\theta_{23}=48^{\circ}-51^{\circ}\right)$. Right panel : $P_{\mu e}$ vs $\varepsilon_{e e}$ for DUNE. Here the bands correspond to LO.

The left plot of fig. (2), shows the hierarchy $\chi^{2}$ vs $\varepsilon_{e e}$ (test) of the DUNE experiment (scheduled run of $5 v+5 \bar{v}$ ) assuming the presence of non-zero diagonal NSI in nature. The $\chi^{2}$ is obtained by considering $\mathrm{NH}$ to be the true hierarchy while keeping true $\delta_{C P}=-\pi / 2$ and testing it with the opposite hierarchy - IH. The different curves - black, red and yellow correspond to different true values of $\varepsilon_{e e}=0,-1,1$ respectively. In the case where $\varepsilon_{e e}=0,1$ (black curve and yellow curve) we can see that there is no hierarchy sensitivity when test $\varepsilon_{e e} \in[-4,4]$, due to the presence of the generalized hierarchy degeneracy of the form $\varepsilon_{e e} \rightarrow-\varepsilon_{e e}-2$. However, when true $\varepsilon_{e e}=-1$ the corresponding degenerate value of $\varepsilon_{e e}$ is also -1 and this can't be removed even when $\varepsilon_{e e}$ is estimated precisely around this value.

The right plot of fig. (2) shows the hierarchy $\chi^{2}$ vs $\varepsilon_{e e}$ (test) of DUNE (for a run of $10 v+0 \bar{v}$ ) plotted assuming the presence of non-zero diagonal and off-diagonal NSI in nature. In this plot the black curve corresponds to the case where true $\varepsilon_{e e}=-1$ showing that there is no hierarchy sensitivity in the absence of off-diagonal NSI due to the presence of the intrinsic hierarchy degeneracy. Thus the confirmation of hierarchy sensitivity at DUNE with $3 \sigma$ significance would eliminate the parameter space of $-1.6<\varepsilon_{e e}<0.8$. 

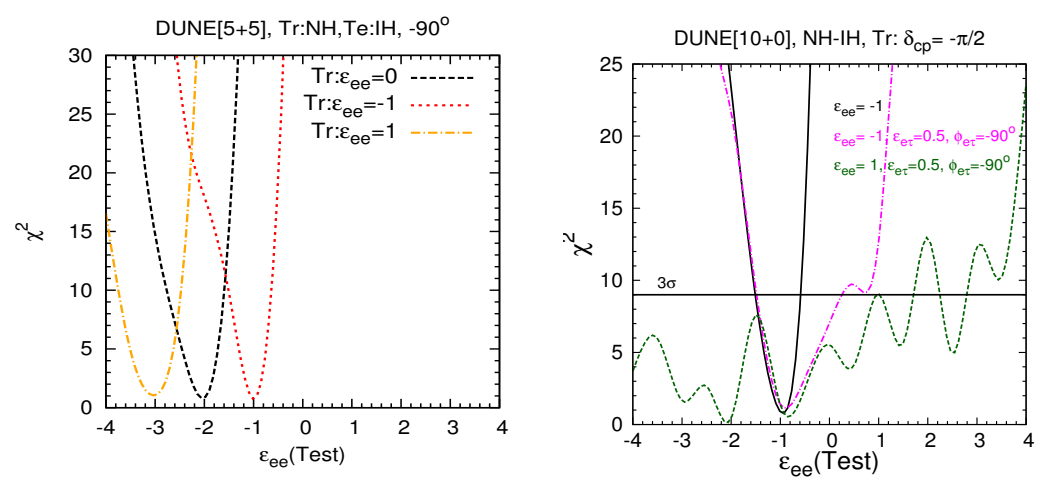

Figure 2: Hierarchy $\chi^{2}$ vs $\varepsilon_{e e}$ (test). Left panel : Hierarchy sensitivity for the scheduled run of $5 v+5 \bar{v}$, true $\delta_{C P}=-\pi / 2$ and test hierarchy - IH. Right panel : Hierarchy sensitivity for a run of $10 v+0 \bar{v}$, true $\delta_{C P}=-\pi / 2$ and marginalized over test hierarchy.

For the case of true $\varepsilon_{e e}=-1, \varepsilon_{e \tau}=0.5$ and $\phi_{e \tau}=-90^{\circ}$, shown by the magenta curve, there is no hierarchy sensitivity and the global minimum occurs at $\varepsilon_{e e}=-0.8$. This is because the presence of the non-zero off-diagonal NSI parameter $\varepsilon_{e \tau}$ would only shift the intrinsic hierarchy degeneracy from - 1 to some other value. This is discussed in detail in fig. (3) of ref. [6].

Lastly, when we assume the true $\varepsilon_{e e}=1\left(\varepsilon_{e e} \neq-1\right)$ and true $\varepsilon_{e \tau}=0.5$ and $\phi_{e \tau}=-90^{\circ}$, there exists a global minimum at $\varepsilon_{e e}=-2$ which exihibits more generalized hierarchy degeneracy of the form $P^{N H}\left(\varepsilon_{e e}, \delta_{C P}, \varepsilon_{e \tau}, \phi_{e \tau}\right)=P^{I H}\left(\varepsilon_{e e}^{\prime}, \delta_{C P}^{\prime}, \varepsilon_{e \tau}^{\prime}, \phi_{e \tau}^{\prime}\right)$. However DUNE can achieve a $2 \sigma$ sensitivity if the parameter region $\varepsilon_{e e}<0.8$ is excluded.

Implications of $\varepsilon_{e e}^{\oplus}=-1$ on the MSW effect in the SUN : Neutrinos undergo resonant flavor transitions while they propagate from the core to the surface of the Sun through the MikheyevSmirnov-Wolfenstein (MSW) effect. The effective hamiltonian in the presence of non-standard neutrino interactions can be written under the assumption of one-mass scale dominance (OMSD) as

$$
H_{e f f}=U_{12}\left[\begin{array}{cc}
0 & 0 \\
0 & \Delta_{21}
\end{array}\right] U_{12}^{\dagger}+\sum_{f} V_{f}\left[\begin{array}{ccc}
c_{13}^{2} \delta_{e f}-\varepsilon_{D}^{f} & \varepsilon_{N}^{f} \\
\varepsilon_{N}^{f *} & \varepsilon_{D}^{f}
\end{array}\right]
$$

where $\Delta_{21} \equiv \Delta m_{21}^{2} /(4 E), U_{12}=\left[\begin{array}{cc}c_{12} & s_{12} \\ -s_{12} & c_{12}\end{array}\right]$ and $V_{f}=V_{f}(r) \equiv \sqrt{2} G_{F} N_{f}(r)$.

The diagonal $\varepsilon_{D}^{f}$ (real) and off-diagonal $\varepsilon_{N}^{f}$ (complex) NSI paramaters can be written in terms of $\varepsilon_{\alpha \beta}$ as $[7,8]$,

$$
\begin{aligned}
\varepsilon_{D}^{f}= & c_{13} s_{13} \operatorname{Re}\left[e^{i \delta_{C P}}\left(s_{23} \varepsilon_{e \mu}^{f}+c_{23} \varepsilon_{e \tau}^{f}\right)\right] \\
- & \left(1+s_{13}^{2}\right) c_{23} s_{23} \operatorname{Re}\left(\varepsilon_{\mu \tau}^{f}\right) \\
- & \frac{c_{13}^{2}}{2}\left(\varepsilon_{e e}^{f}-\varepsilon_{\mu \mu}^{f}\right)+\frac{s_{23}^{2}-s_{13}^{2} c_{23}^{2}}{2}\left(\varepsilon_{\tau \tau}^{f}-\varepsilon_{\mu \mu}^{f}\right), \\
\varepsilon_{N}^{f}= & c_{13}\left(c_{23} \varepsilon_{e \mu}^{f}-s_{23} \varepsilon_{e \tau}^{f}\right)+s_{13} e^{-i \delta_{C P}} \times \\
& {\left[s_{23}^{2} \varepsilon_{\mu \tau}^{f}-c_{23}^{2} \varepsilon_{\mu \tau}^{f *}+c_{23} s_{23}\left(\varepsilon_{\tau \tau}^{f}-\varepsilon_{\mu \mu}^{f}\right)\right] . }
\end{aligned}
$$


The survival probability of solar neutrinos is obtained by :

$$
P_{e e}=s_{13}^{4}+c_{13}^{4} P_{e f f}
$$

where $P_{\text {eff }}$ is the survival probability obtained by solving the neutrino evolution equation in the effective 2-flavor system. The effective mixing angle $\theta_{M}$ in matter can be obtained by diagonalizing the effective hamiltonian in eq. (5):

$$
\tan 2 \theta_{M}=\frac{2\left(\Delta_{21} \sin 2 \theta_{12}+\sum_{f} V_{f} \varepsilon_{N}^{f}\right)}{2 \Delta_{21} \cos 2 \theta_{12}-\sum_{f} V_{f}\left(c_{13}^{2} \delta_{e f}-2 \varepsilon_{D}^{f}\right)}
$$

Note that if $\varepsilon_{\alpha \beta}=0$ i.e. when NSIs are absent then $\tan \left(2 \theta_{M}\right)$ reduces to its standard form.

When we consider the presence of only non-zero diagonal NSI and assume $\varepsilon_{e e}^{\oplus}=-1$ (for instance : $\varepsilon_{e e}^{u}=-1 / 3$ and $\varepsilon_{e e}^{d}=0 ; 1 \sigma$ allowed region of CHARM results) we found

$$
\begin{aligned}
\tan 2 \theta_{M} & =\frac{2 \Delta_{21} \sin 2 \theta_{12}}{2 \Delta_{21} \cos 2 \theta_{12}-V_{e} c_{13}^{2} / 6}, \\
& =\frac{\tan 2 \theta}{1-\frac{N_{e}}{N_{e, \text { res }}}}
\end{aligned}
$$

Thus, when the condition

$$
\Delta_{21} \cos 2 \theta_{12}=V_{e} c_{13}^{2} / 12
$$

is satisfied MSW resonance occurs in Sun and the corresponding electron density is

$$
N_{e, r e s}=\frac{3 \sqrt{2} \Delta m_{21}^{2} \cos 2 \theta_{12}}{G_{F} E c_{13}^{2}} .
$$

For the above case assuming $\varepsilon_{e e}^{\oplus}=-1$, we observed that MSW resonance occurs in the Sun for neutrinos of energy $E \geq 7.2 \mathrm{MeV}$. For $\Delta m_{21}^{2} \sim 4 \times 10^{-6} \mathrm{eV}^{2}$ and $\sin ^{2} \theta_{12}=0.3$ i.e. the Large Mixing Angle (LMA) solution, the matter mixing angle and the corresponding survival probability are found to be $\cos 2 \theta_{M} \sim-1$ and $P_{e e} \sim 0.3$ respectively.

In conclusion, we have explored various degeneracies introduced by non-zero NSI parameters and their effect on the hierarchy sensitivity of the DUNE baseline. We elaborated on an interesting case by assuming $\varepsilon_{e e}^{\oplus}=-1$ and $\delta_{C P}=-\pi / 2$. This leads to an intrinsic hierarchy degeneracy independent of the baseline and the energy. Additionally we have discussed the implications of $\varepsilon_{e e}^{\oplus}=-1$ on the MSW effect of the Sun.

\section{References}

[1] L. Wolfenstein, Phys. Rev. D 17, 2369 (1978).

[2] T. Ohlsson, Rept. Prog. Phys. 76, 044201 (2013).

[3] P. Huber, M. Lindner and W. Winter, Comput. Phys. Commun. 167, 195 (2005); P. Huber, et al., Comput. Phys. Commun. 177, 432 (2007).

[4] J. Kopp, Int. J. Mod. Phys. C 19, 523 (2008); J. Kopp, et al., Phys. Rev. D 77, 013007 (2008).

[5] P. Coloma and T. Schwetz, Phys. Rev. D 94, 055005 (2016); J. Liao, D. Marfatia and K. Whisnant, Phys. Rev. D 93, 093016 (2016). 
[6] Deepthi, K. N. and Goswami, Srubabati and Nath, Newton, Phys. Rev. D 96, 075023 (2017).

[7] Kuo, Tzee-Ke and Pantaleone, James T. Phys. Lett. 57, 1805-1808 (1986).

[8] Gonzalez-Garcia, M. C. and Maltoni, Michele, JHEP 1309, 152, (2013). 